\title{
Relationships between serum procalcitonin level, severity and different stresses of non-septic critically ill patients
}

\author{
Juncheng Shi, Qiankun Shi*, Shoutao Yuan, Wenhao Zhang \\ Department of Intensive Care Medicine, Nanjing First Hospital, Nanjing, Jiangsu Province, P. R. \\ China
}

\begin{abstract}
Background: To explore the relationships between serum procalcitonin (PCT) level, severity and different stresses of non-septic critically ill patients. Materials and Methods: Patients were divided into traumatic stress, stroke-induced stress and non-infectious inflammatory stress groups. According to 28-day prognosis, they were divided into survival and death groups. The factors affecting prognosis were studied by multivariate logistic regression analysis. Results: PCT level was significantly positively correlated with Acute Physiology and Chronic Health Evaluation II (APACHE II) and sequential organ failure assessment (SOFA) scores $(P=0.001)$. The PCT level and abnormality rate of the traumatic stress group significantly exceeded those of other groups $(P<0.05)$. The APACHE II score, SOFA score and 28-day mortality rate of traumatic stress and stroke-induced stress groups significantly exceeded those of the non-infectious inflammatory stress group $(P<0.05)$. The PCT level, APACHE II score and SOFA score of the death group significantly surpassed those of the survival group $(P<0.05)$. With rising PCT level, APACHE II score, SOFA score and 28-day mortality rate all increased, with significant intergroup differences $(P<0.01)$. Multivariate logistic analysis showed that serum PCT level, APACHE II score and SOFA score were independent risk factors for prognosis. The area under ROC curve for prognosis evaluated by PCT level was $0.797(95 \% C I=$ 0.710 0.878, P=0.000). At a $4.3 \mu \mathrm{g} / \mathrm{L}$ cut-off, the sensitivity and specificity for predicting 28-day mortality were $87.4 \%$ and $78.1 \%$, respectively. Conclusion: The serum PCT level of non-septic critically ill patient was positively correlated with severity, which was more likely elevated by traumatic stress than other stresses.
\end{abstract}

Keywors: procalcitonin, non-sepsis, stress, severity, prognosis

Received: 23 ${ }^{\text {rd }}$ July 2019; Accepted: $3^{\text {rd }}$ November 2019; Published: $21^{\text {st }}$ November 2019

\section{Introduction}

As a peptide precursor of calcitonin, procalcitonin (PCT) is mainly produced by thyroid $\mathrm{C}$ cells, consisting of 116 amino acid residues. It is a glycoprotein without hormone activity. Increased serum PCT levels of patients with lung cancer suggest that endocrine cells of the pulmonary nerve also secrete PCT (1). Besides, the serum PCT level is significantly elevated in septic patients receiving thyroidectomy, but that of calcitonin hardly changes (2). Therefore, not only thyroid C cells synthesize and secrete PCT.

*Corresponding author: Qiankun Shi, Department of Intensive Care Medicine, Nanjing First Hospital, No. 68 Changle Road, Nanjing 210006, Jiangsu Province, P. R. China. E-mail: shiqiankunnfh@yeah.net 
Many types of cells in various tissues of human body can induce the release of PCT under inflammatory stimuli (3). It is well-known that the PCT level increases upon sepsis, especially in the case of bacterial infections, being positively correlated with bacterial load and severity of infection $(4,5)$. Moreover, the PCT level is positively correlated with the Acute Physiology and Chronic Health Evaluation II (APACHE II) score of patients with sepsis, reflecting disease severity and prognosis $(6,7)$. Thus, PCT is commonly used as a specific biomarker for diagnosing bacterial infections. Many non-infectious factors, such as severe trauma, major surgery, cardiogenic shock and some autoimmune diseases, can also produce inflammatory mediators and cytotoxins in human body, thereby raising the serum PCT level (8). However, the relationships between serum PCT level, severity and prognosis of non-infectious diseases have seldom been reported hitherto. Meanwhile, non-infectious critically ill patients may suffer from traumatic stress, stroke-induced stress or systemic inflammatory response syndrome which can increase the serum PCT level by inducing the release of inflammatory factors (8). Nevertheless, the relationships between different stresses and PCT level remain unknown. The aim of this study was to explore the possible relationships between the PCT level, disease severity and different stresses of non-septic critically ill patients, as well as the predictive value for prognosis.

\section{Materials and Methods}

\section{Subjects}

This study has been approved by the ethics committee of our hospital (approval No. 20150731042). A total of 188 non-septic critically ill patients admitted to our hospital from August 2015 to December 2017 were retrospectively analyzed. Inclusion criteria: Critically ill patients without sepsis; aged $>18$ years old; with written informed consent. Exclusion criteria: Patients with ICU stay length of $<3 \mathrm{~d}$; in accordance with the diagnostic criteria of sepsis in the 2012 International Guidelines for the Treatment of Severe Sepsis and Septic Shock (9) within $24 \mathrm{~h}$ after ICU admission; with suspected clinical infections within $3 \mathrm{~d}$ after ICU admission.

From each included patient, $5 \mathrm{~mL}$ of venous blood was collected and centrifuged within 30 min to obtain the serum that was then stored in a $4^{\circ} \mathrm{C}$ refrigerator prior to use. PCT level was measured within $24 \mathrm{~h}$ by electrochemiluminescence assay using a kit purchased from Roche Diagnostics (Shanghai, China). The 188 patients comprised 80 males and 108 females who were aged 22-80 years old, $(55.4 \pm 8.3)$ on average. The serum PCT level was $4.53(1.20,9.10) \mu \mathrm{g} / \mathrm{L}$, the APACHE II score was $(12.3 \pm 7.2)$ points, and the SOFA score was $5.0(3.0,8.0)$ points. After 28 days, 156 cases survived and 32 died.

\section{Grouping}

The enrolled patients were divided into a traumatic stress group $(\mathrm{n}=56)$, a stroke-induced stress group ( $n=60)$ and a non-infectious inflammatory stress group $(n=72)$ according to primary diseases. The traumatic stress group included 30 cases of multiple traumas and 26 cases after cardiopulmonary resuscitation. The stroke-induced stress group included 40 cases of hemorrhagic stroke and 20 cases of ischemic stroke. The non-infectious inflammatory stress group included 20 cases of severe acute pancreatitis, 20 cases of myasthenia gravis and 32 cases of autoimmune diseases (Table 1).

In the meantime, the enrolled patients were divided into a normal group (PCT $<0.5 \mu \mathrm{g} / \mathrm{L}$ ), a low-level group $(0.5 \mu \mathrm{g} / \mathrm{L} \leq \mathrm{PCT}<2 \mu \mathrm{g} / \mathrm{L})$, a middle-level group $(2 \mu \mathrm{g} / \mathrm{L} \leq \mathrm{PCT}<5 \mu \mathrm{g} / \mathrm{L})$ and a high-level group (PCT $\geq 5 \mu \mathrm{g} / \mathrm{L}$ ) according to serum PCT level. 
Table 1. Grouping and case descriptions

\begin{tabular}{lc}
\hline Group & $\begin{array}{c}\text { Case } \\
\text { number }\end{array}$ \\
\hline Traumatic stress & 56 \\
\hline Multiple traumas & 30 \\
\hline After cardiopulmonary resuscitation & 26 \\
\hline Stroke-induced stress & 60 \\
\hline Hemorrhagic stroke & 40 \\
\hline Ischemic stroke & 20 \\
\hline Non-infectious inflammatory stress & 72 \\
\hline Severe acute pancreatitis & 20 \\
\hline Myasthenia gravis & 20 \\
\hline Autoimmune diseases & 32 \\
\hline
\end{tabular}

\section{Data collection and evaluation}

The gender, age, blood cell count, liver and kidney functions, coagulation function, arterial blood gas analysis, requirement of surgery, mechanical ventilation index, vasoactive drug dose and 28-day mortality rate of patients were recorded. The APACHE II score and sequential organ failure assessment (SOFA) score were calculated according to clinical and laboratory indices within $24 \mathrm{~h}$ after admission into ICU. The PCT levels of patients with different stresses were compared. The prognosis of patients with different PCT levels was observed. The receiver operating characteristic (ROC) curve was plotted to evaluate the predictive value of PCT level for 28-day prognosis.

\section{Statistical analysis}

All data were statistically analyzed by SPSS 19.0 software. The normally distributed categorical data were expressed as mean \pm standard deviation $(\mathrm{x} \pm \mathrm{s}$ ). Intergroup comparisons were performed by the $t$ test, and multigroup comparisons were conducted with one-way analysis of variance. The non-normally distributed categorical data were represented as median (quartile, $\mathrm{M}$ (QL, QU)). Intergroup comparisons were carried out with the Kruskal-Wallis U test, and multigroup comparisons were performed by us- ing the Kruskal-Wallis $\mathrm{H}$ test. Variables were subjected to Pearson's or Spearman's correlation analysis. The factors affecting prognosis were studied by multivariate logistic regression analysis. The area under ROC curve using PCT level was calculated. $\mathrm{P}<0.05$ was considered statistically significant.

\section{Results}

\section{Correlations between serum PCT level and APACHE II and SOFA scores}

Serum PCT level was significantly positively correlated with APACHE II and SOFA scores $\left(r_{1}\right.$ $=0.679, \mathrm{r}_{2}=0.787$, both $\left.\mathrm{P}=0.000\right)$.

PCT level, disease severity and 28-day mortality rate of patients under different stresses

The traumatic stress, stroke-induced stress and non-infectious inflammatory stress groups had similar gender ratio, age and body mass index (BMI) $(\mathrm{P}>0.05)$. The serum PCT level and abnormality rate of the traumatic stress group were significantly higher than those of stroke-induced stress and non-infectious inflammatory stress groups $(\mathrm{P}<0.05)$. The APACHE II score, SOFA score and 28-day mortality rate of traumatic stress and stroke-induced stress groups significantly exceeded those of the non-infectious inflammatory stress group $(\mathrm{P}<0.05)$ (Table 2$)$.

\section{PCT level and disease severity of patients with different prognoses}

The death and survival groups had similar gender ratio, age and $\mathrm{BMI}(\mathrm{P}>0.05)$. The serum $\mathrm{PCT}$ level, APACHE II score and SOFA score of the death group significantly surpassed those of the survival group $(\mathrm{P}<0.05)$ (Table 3$)$.

\section{Disease severity and 28-day mortality rate of patients with different PCT levels}

There were significantly more males in the middle-level group than in the normal, low-level 


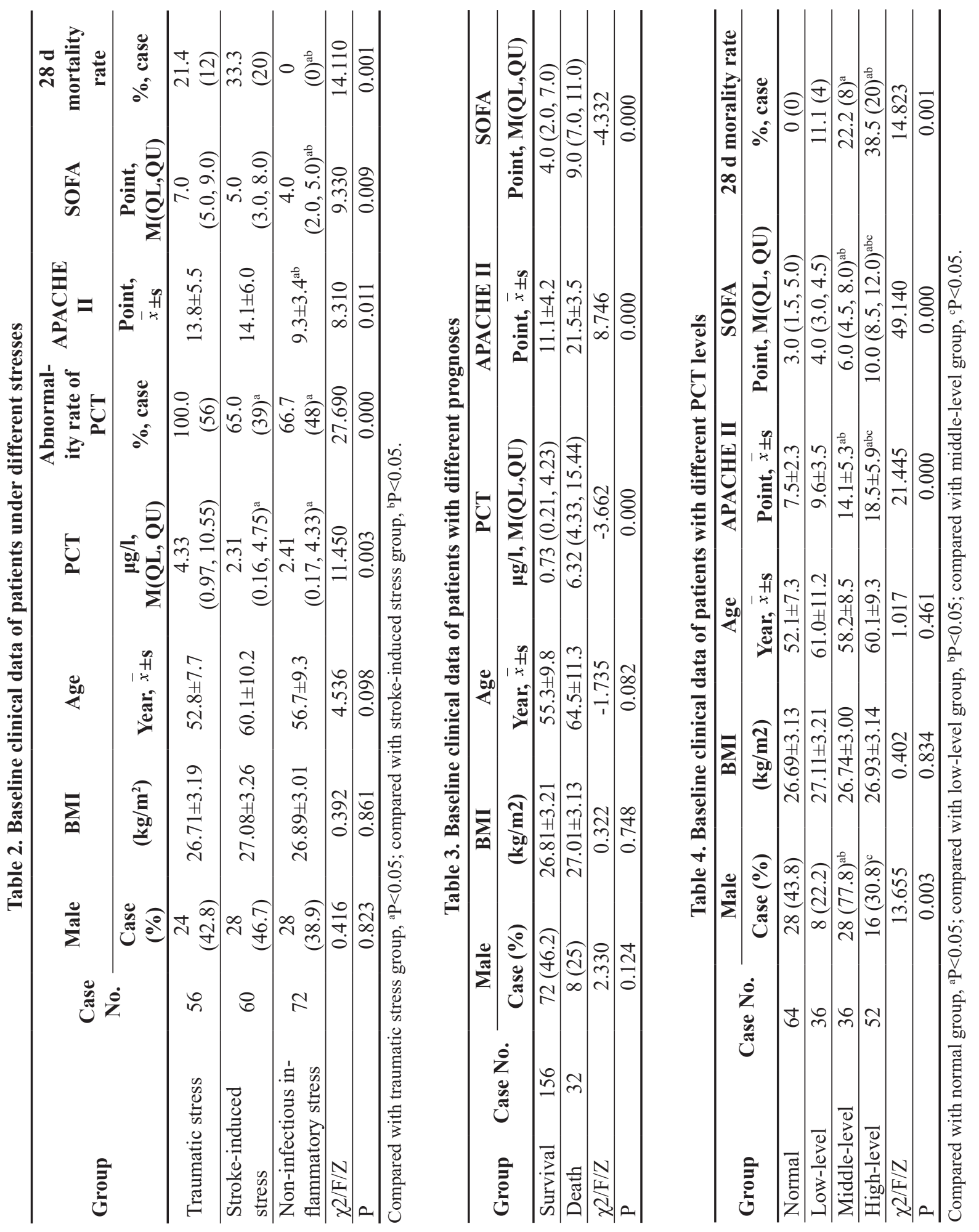


and high-level groups $(\mathrm{P}<0.05)$. However, the levels did not significantly differ depending on age or BMI $(\mathrm{P}>0.05)$. With increasing PCT level, APACHE II score, SOFA score and 28day mortality rate all increased, with significant differences among groups $(\mathrm{P}<0.01)$. The middle-level group had significantly higher APACHE II score, SOFA score and 28-day mortality rate than those of normal and low-level groups, and the values of the high-level group significantly exceeded those of the other three groups $(\mathrm{P}<0.05)$ (Table 4).

\section{Multivariate logistic regression analysis of factors affecting prognosis}

Multivariate logistic regression analysis was conducted by using serum PCT level, APACHE II score and SOFA score as the independent variables and 28-day prognosis as the dependent variable. Table 5 shows that serum PCT level, APACHE II score and SOFA score are independent risk factors for the prognosis of non-septic critically ill patients (Table 5).

\section{Prognostic value of serum PCT level}

The area under ROC curve for prognosis evaluated by PCT level was $0.797(95 \% \mathrm{CI}=$ $0.710 \sim 0.878, \mathrm{P}=0.000)$. When the cut-off value was $4.3 \mu \mathrm{g} / \mathrm{L}$, the sensitivity and specificity for predicting 28 -day mortality rate were $87.4 \%$ and $78.1 \%$, respectively (Figure 1).

\section{Discussion}

PCT secretion is largely stimulated by inflammatory mediators and bacterial toxins (10). Currently, the relationships between PCT and sepsis have been extensively studied. The APACHE II score is commonly used to assess the severity of critically ill patients in ICU. It is also an effective predictor for mortality (11). SOFA is also one of the commonly used scoring systems in ICU for organ failure assessment. This study explored the relationships between PCT levels and APACHE II and SOFA scores of non-septic critically ill patients. The serum PCT level was positively correlated with APACHE II and SOFA scores. Since APACHE II and SOFA scores can predict the severity of critically ill patients and reflect their prognosis, the serum PCT level indirectly indicates the disease severity. Similarly, the serum PCT level has been positively correlated with the severity of acute pancreatitis $(12,13)$. Injecting PCT into septic animals evidently increased the mortality rate, whereas use of PCT antiserum raised the survival rate, suggesting that elevation of PCT level was linked to increase of mortality rate (14). In addition, PCT

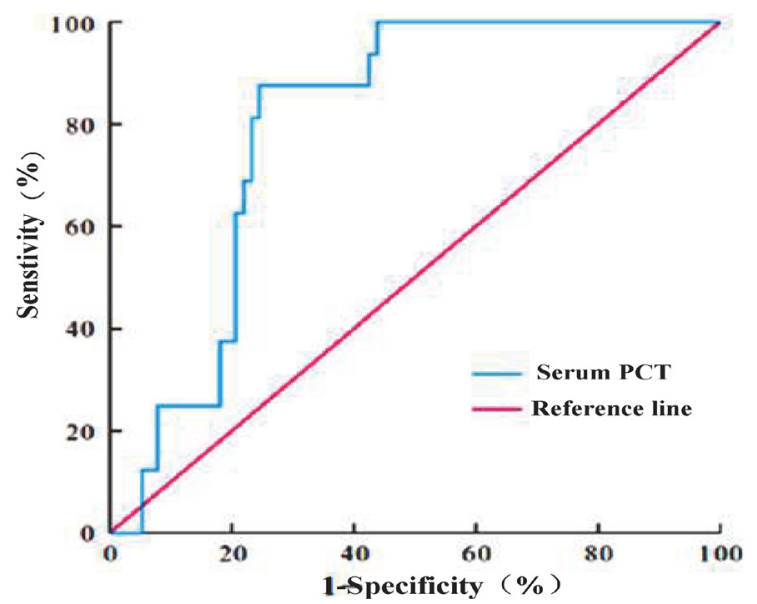

Fig. 1. ROC curve for prognostic value of serum PCT level

Table 5. Multivariate logistic regression analysis of factors affecting prognosis

\begin{tabular}{lcccccc}
\hline Factor & $\begin{array}{c}\text { Regression } \\
\text { coefficient }\end{array}$ & $\begin{array}{c}\text { Standard } \\
\text { error }\end{array}$ & Wald value & P value & OR & 95\%CI \\
\hline Serum PCT level & 2.045 & 0.912 & 5.004 & 0.021 & 3.226 & $2.445 \sim 6.892$ \\
\hline APACHE II score & 3.145 & 0.827 & 7.654 & 0.020 & 1.325 & $1.064 \sim 1.435$ \\
\hline SOFA score & 2.184 & 0.454 & 4.417 & 0.011 & 1.378 & $1.083 \sim 2.472$ \\
\hline
\end{tabular}


was an effective index for the early diagnosis of sepsis, and dynamic monitoring of its level can predict the severity and prognosis of this disease, so PCT level was an independent risk factor for predicting the 28-day survival (15). We herein found that in non-septic critically ill patients, the 28-day mortality rate of the high-level group was significantly higher than those of other groups. Multivariate logistic analysis showed that serum PCT level, APACHE II score and SOFA score were independent risk factors for the prognosis of non-septic critically ill patients. ROC analysis revealed that when PCT $>4.2 \mu \mathrm{g} / \mathrm{L}$, the sensitivity of predicting 28-day mortality was augmented. Therefore, PCT level may be an independent predictor for the death risk of critically ill patients.

Up to now, the relationships between PCT levels and different stresses are still elusive. In this study, patients were grouped according to the type of stress to explore the possible relationship between PCT level and stress type. The serum PCT level and abnormality rate of the traumatic stress group were significantly higher than those of stroke-induced stress and non-infectious inflammatory stress groups ( $\mathrm{P}<0.05)$. Additionally, the APACHE II score, SOFA score and 28-day mortality rate of traumatic stress and stroke-induced stress groups significantly exceeded those of the non-infectious inflammatory stress group $(\mathrm{P}<0.05)$. Trauma can trigger systemic inflammatory response. Damaged cells and tissues as well as stressed cells release large amounts of inflammatory factors to cause immune responses (16). Thus, PCT production can be stimulated by traumatic stress.

In this study, the severity of the non-infectious inflammatory stress group was lower than that of the stroke-induced stress group, but the two groups had similar PCT levels, so we postulated that non-infectious inflammatory stress elevated the level more easily. Zhao et al. reported that PCT level increased in non-infectious SIRS patients, as a predictive factor for prognosis (17). Tuzun et al. also found that PCT level significantly increased in the acute phase of ulcerative colitis, being associated with the control of disease progression (18). Herein, the non-infectious inflammatory stress group included cases with severe acute pancreatitis, myasthenia gravis and autoimmune diseases. Upon acute pancreatitis, acinar cell damage can lead to inflammatory cell aggregation and promote the production and release of cytokines and other inflammatory mediators, and the disease severity may be determined by this process (19). Therefore, PCT level elevation in the early stage of acute pancreatitis can be attributed to the release of considerable inflammatory factors, but further increase with disease progression is linked to secondary infection caused by pancreatic necrosis and abscess (20,21). Although PCT level is commonly used to diagnose the secondary infection of autoimmune diseases, slight increase has also been observed in the critical phase without infection. ${ }^{22}$ Furthermore, different stress groups had similar gender ratio, age and $\mathrm{BMI}(\mathrm{P}>0.05)$. Nevertheless, there were gender differences among different PCT level groups, probably being linked to the small sample size. In-depth studies with larger sample sizes on ongoing in our group.

In summary, the serum PCT levels of non-septic critically ill patients were positively correlated with disease severity, showing predictive value for prognosis. PCT level was more likely elevated by traumatic stress than stroke-induced stress and non-infectious inflammatory stress. In future studies, we will dynamically monitor the effects of PCT level changes on disease severity and prognosis within $24 \mathrm{~h}$ after ICU admission, and clarify the mechanism for the differences between PCT levels under various stresses. 


\author{
Abbreviations \\ APACHE II - Acute Physiology and Chronic \\ Health Evaluation II \\ BMI - body mass index \\ $\mathrm{PCT}$ - procalcitonin \\ ROC - receiver operating characteristic \\ SOFA - sequential organ failure assessment
}

\section{Conflict of interest}

There was no conflict in this work.

\section{References}

1. Avrillon V, Locatelli-Sanchez M, Folliet L, Carbonnaux M, Perino E, Fossard G, et al. Lung cancer may increase serum procalcitonin level. Infect Disord Drug Targets. 2015;15(1):57-63. DOI: 10.2174/1871526515 666150320162950

2. Kaczka K, Mikosiński S, Fendler W, Celnik A, Pomorski L. Calcitonin and procalcitonin in patients with medullary thyroid cancer or bacterial infection. Adv Clin Exp Med. 2012;21(2):169-78.

3. Rami D, La Bianca M, Agostinis C, Zauli G, Radillo O, Bulla R. The first trimester gravid serum regulates procalcitonin expression in human macrophages skewing their phenotype in vitro. Mediators Inflamm. 2014;2014:248963. DOI: 10.1155/2014/248963

4. Fung AWS, Beriault D, Diamandis EP, Burnham $\mathrm{CD}$, Dorman $\mathrm{T}$, Downing $\mathrm{M}$, et al. The Role of Procalcitonin in Diagnosis of Sepsis and Antibiotic Stewardship: Opportunities and Challenges. Clin Chem. 2017;63(9):1436-41. DOI: 10.1373/ clinchem.2017.272294

5. Schuetz P, Bretscher C, Bernasconi L, Mueller B. Overview of procalcitonin assays and procalcitonin-guided protocols for the management of patients with infections and sepsis. Expert Rev Mol Diagn. 2017;17(6):593601. DOI: 10.1080/14737159.2017.1324299

6. Jiang L, Feng B, Gao D, Zhang Y. Plasma concentrations of copeptin, C-reactive protein and procalcitonin are positively correlated with APACHE II scores in patients with sepsis. J Int Med Res. 2015;43(2):188-95. DOI: $10.1177 / 0300060514561136$

7. Jain S, Sinha S, Sharma SK, Samantaray JC, Aggrawal P, Vikram NK, et al. Procalcitonin as a prognostic marker for sepsis: a prospective observational study. BMC Res Notes. 2014;7(1):458. DOI: 10.1186/17560500-7-458

8. Anand D, Das S, Bhargava S, Srivastava LM, Garg A, Tyagi N, et al. Procalcitonin as a rapid diagnostic biomarker to differentiate between culture-negative bacterial sepsis and systemic inflammatory response syndrome: a prospective, observational, cohort study. J Crit Care. 2015;30(1):218.e7-12. DOI: 10.1016/j. jcrc.2014.08.017

9. Dellinger RP, Levy MM, Rhodes A, Annane D, Gerlach H, Opal SM, et al. Surviving Sepsis Campaign: international guidelines for management of severe sepsis and septic shock, 2012. Crit Care Med. 2013;41(2):580637. DOI: 10.1097/CCM.0b013e31827e83af

10. Schuetz P, Wirz Y, Sager R, Christ-Crain M, Stolz D, Tamm M, et al. Effect of procalcitonin-guided antibiotic treatment on mortality in acute respiratory infections: a patient level meta-analysis. Lancet Infect Dis. 2018;18(1):95-107. DOI: 10.1016/S14733099(17)30592-3

11. Quach S, Hennessy DA, Faris P, Fong A, Quan H, Doig C. A comparison between the APACHE II and Charlson Index Score for predicting hospital mortality in critically ill patients. BMC Health Serv Res. 2009;9:129-36. DOI: 10.1186/1472-6963-9-129

12. Yang CJ, Chen J, Phillips AR, Windsor JA, Petrov MS. Predictors of severe and critical acute pancreatitis: a systematic review. Dig Liver Dis. 2014;46(5):446-51. DOI: 10.1016/j.dld.2014.01.158

13. Staubli SM, Oertli D, Nebiker CA. Laboratory markers predicting severity of acute pancreatitis. Crit Rev Clin Lab Sci. 2015;52(6):273-83. DOI: 10.3109/10408363.2015.1051659

14. Becker KL, Snider R, Nylen ES. Procalcitonin in sepsis and systemic inflammation: a harmful biomarker and a therapeutic targe. Br J Pharmacol. 2010;159(2):253-64. DOI: 10.1111/j.1476-5381.2009.00433.x

15. Ryu JA, Yang JH, Lee D, Park CM, Suh GY, Jeon K, et al. Clinical Usefulness of Procalcitonin and C-Reactive Protein as Outcome Predictors in Critically Ill Patients with Severe Sepsis and Septic Shock. PLoS One. 2015;10(9):e0138150. DOI: 10.1371/journal. pone. 0138150

16. Trásy D, Molnár Z. Procalcitonin - Assisted Antibiotic Strategy in Sepsis. EJIFCC. 2017;28(2):104-13.

17. Zhao JJ, Lou XL, Chen HW, Zhu FT, Hou YQ. Diagnostic value of decoy receptor 3 combined with procalcitonin and soluble urokinase-type plasminogen activator receptor for sepsis. Cell Mol Biol Lett. 2018;23:2231. DOI: $10.1186 / \mathrm{s} 11658-018-0087-\mathrm{z}$

18. Tuzun A, Uygun A, Yesilova Z, Ozel AM, Erdil A, Yaman $H$, et al. Leptin levels in the acute stage of ulcerative colitis. J Gastroenterol Hepatol. 2004;19(4):42932. DOI: 10.1111/j.1440-1746.2003.03300.x

19. Chooklin S, Hranat O. Intraarterial therapy, interleukins and oxidative stress parameters in acute pancreatitis patients. Pancreatology. 2013;13(2):e20. DOI: 10.1016/j. pan.2012.12.124

20. Liu HH, Guo JB, Geng Y, Su L. Procalcitonin: present and future. Ir J Med Sci. 2015;184(3):597-605. DOI: 
10.1007/s11845-015-1327-0

21. Huang HL, Nie X, Cai B, Tang JT, He Y, Miao Q, et al. Procalcitonin levels predict acute kidney injury and prognosis in acute pancreatitis: a prospective study. PLoS One. 2013;8(12):e82250. DOI: 10.1371/journal. pone. 0082250
22. Wu JY, Lee SH, Shen CJ, Hsieh YC, Yo PH, Cheng HY, et al. Use of serum procalcitonin to detect bacterial infection in patients with autoimmune diseases: a systematic review and meta-analysis. Arthritis Rheum. 2014;64(9):3034-42. DOI: 10.1002/art.34512 